\title{
Substitute fluid examinations for liquid manure
}

\author{
Kevin Schrader ${ }^{1, *}$, Marco Riedel $^{1}$ and Helmut Eichert $^{1}$ \\ ${ }^{1}$ University of Applied Sciences Zwickau, Department of Automotive Engineering, Dr.-Friedrichs-Ring 2A, 08056 Zwickau (Germany)
}

\begin{abstract}
For the farming industry it is essential to use liquid manure as natural fertilizer. Through new agricultural regulation 2015 in Germany the industry must develop new liquid manure spreader systems because the ammonia and methane emission are limited. In a research project the University of Applied Sciences Zwickau and some other industry partners will develop such a new innovative liquid manure spreader. The new liquid manure spreader should use pulsating air to distribute the liquid manure exactly. The pulsating air, which flows through the pipelines, should be analysed at a test station. For examinations at this test station it is important to find another substitute fluid because liquid manure smells strong, is not transparent and is also not homogeneous enough for scientific investigations. Furthermore it is important to ensure that the substitute fluid is, like liquid manure, a non-Newtonian fluid. The substitute fluid must be a shear-thinning substance - this means the viscosity decrease at higher shear rate. Many different samples like soap-water-farragoes, jelly-water-farragoes, agar-water-farragoes, soap-ethanol-farragoes and more are, for the project, examined in regard of their physical properties to find the best substitute fluid. The samples are examined at the rotational viscometer for viscosity at various shear rates and then compared with the viscosity values of liquid manure.
\end{abstract}

\section{Introduction}

New regulations from 2015 forced the agricultural industry to optimize the old liquid manure spreader systems. In a research project the University of Applied Sciences Zwickau together with industry partners optimized the liquid manure spreader and examined the impact of pulsating air to distribute the slurry exactly. Therefore it was important to find a substitute fluid for slurry because it smells strong and is not transparent. The substitute fluid has to be transparent because of examinations with the laser Doppler anemometer (short form: LDA). Furthermore the medium and the boundary of the pipe have to be diaphanous [1]. The following article explains the measurement principle of the "cup and bob" rotational viscometer. After that explanation the examination steps for the substitute fluid should be explained.

One kind of the rotational viscometer is the "cub and bob" viscometer, which works with an exact defined volume sample. A "cup and bob" rotational viscometer consists of three important parts. The first part is the cup (1) and the second is the bob or rotor (2). The third important component is the measuring volume (3), which contains the measuring fluid. The measuring substance is filled in the cup. After that the cup is fixed in the rotational viscometer. The component bob immersed in the fluid. The measuring fluid is now located between the cup and the rotor. In figure 1 the setup of a "cup and bob" rotational viscometer is shown.

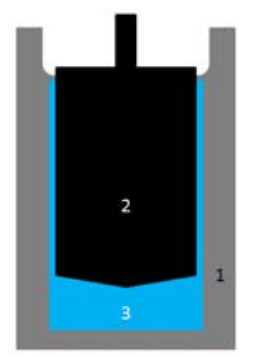

Fig. 1- Setup of a "cup and bob" rotational viscometer, (1) cup, (2) bob/rotor, (3) fluid

There are two different kinds of "cup and bob" rotational viscometers. The first kind is called Couette system. At a Couette system the cup moves and the bob stands still. The other type is named Searle system. The Searle system is characterized by a moving rotor and a cup which is standing still.

The space between the cup and the rotor is called annular gap. This gap forms the measurement volume. The fluid adheres to the boundary of the cup and the rotor. Through the movement of the rotor, the fluid will be moved too. The examining fluid is build up from serval layers. This layers slides on top of each other (laminar flow). Between these layers there is a velocity difference, which is called shear gradient or shear rate.

At the "cup and bob" rotational viscometer it is usually to adjust the angular speed and to measure the torque $\left(\mathrm{M}_{\mathrm{d}}\right)$ at this speed. With the geometric dimensions (surface size - A/ bob radius - r) and the physical sizes of the angular gap (y), force (F) and velocity (v) the dynamic viscosity can be determined. For the

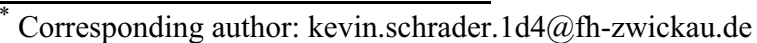


equation (3) it is important to calculate the shear rate and the shear stress. With the force and the dimensions (A and $r$ ), the shear stress can be calculated (equation (1)). Furthermore with the velocity and dimensions of the annular gap it is possible to calculate the shear rate (equation (2)).

Shear Stress:

Shear Rate:

$$
\tau=\frac{M_{d}}{A \cdot r}=\frac{F}{A}
$$

$$
\dot{\gamma}=\frac{\omega}{y \cdot r}=\frac{v}{y}
$$

The following equation is used to calculate the viscosity $(\mu)$ from shear rate $(\dot{\gamma})$ and shear stress $(\tau)$ :

$$
\mu=\frac{\tau}{\dot{\gamma}}
$$

\section{Examinations of cattle and pig manure}

The first task to characterize the mechanical properties of the liquid manure was to analyse a sample in the viscometer. For this study a cattle manure sample was used and examined in a rotational viscometer that was produced by the company Physika. Unfortunately, the measurements led to no plausible values because the fluid contained too much large particle like sand and straw. Therefore to specify slurry publications and reports $([2,3,4])$ from the past years were used. It was important to find articles which contain information about the viscosity in dependent of the shear rate. The next figure shows the values of shear rate in dependent of viscosity for pig manure (shear stress and shear rate were used form the publication [2] - viscosity were calculate of these physical sizes). According to this publication the measurement environment, and so the liquid manure too, had a temperature of $20{ }^{\circ} \mathrm{C}$. The dry matter content (DM) varied from $8-22 \%-D M$.

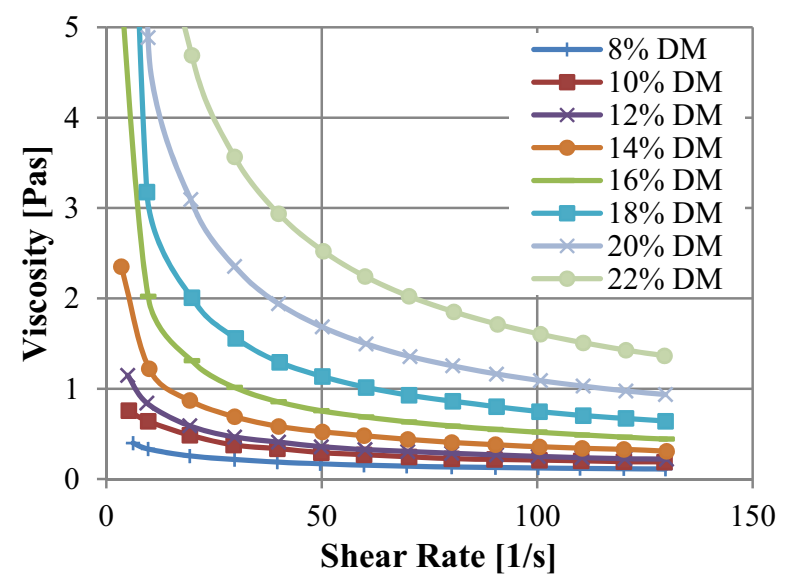

Fig. 2 - Description of the dynamic viscosity in dependent of shear rate from pig manure with several dry matter contents (calculate from source [2])

As in figure 2 shown, the viscosity decrease with increasing shear rate. Fluids which have such a mechanical property are referred as shear-thinning fluids. The shear-thinning liquids and gases belong to the non-Newtonian fluids. This means that the shear rate is not direct proportional to the shear stress. The following substances belong to the representatives of shearthinning fluids:

- ketchup,

- toothpaste,

- paints,

- buttermilk with flakes,

- shampoo.

Also it is apparent from figure 2 that with increasing dry matter content the dynamic viscosity of liquid pig manure increases. The poorer flow behaviour can be attributed to the more viscous phase of the slurry. The dynamic viscosity varies from about 0.50 to $7.00 \mathrm{~Pa} \mathrm{~s}$ at low shear rates of about $0 \ldots 10 \mathrm{l} / \mathrm{s}$. At higher shear rates (from about 75 to $130 \mathrm{l} / \mathrm{s}$ ) the values of the dynamic viscosity are in the range of 0.25 to $1.50 \mathrm{~Pa} \mathrm{~s}$.

In addition to the value of the dry matter content, the temperature can also vary. In figure 3 [4] the viscosity and the shear rate at different temperatures is shown. The slurry temperature was raised from 30 to $60{ }^{\circ} \mathrm{C}$. The dry matter content was at a constant value of $9.1 \%$-DM. Instead of pig manure cattle manure was used for the investigation.

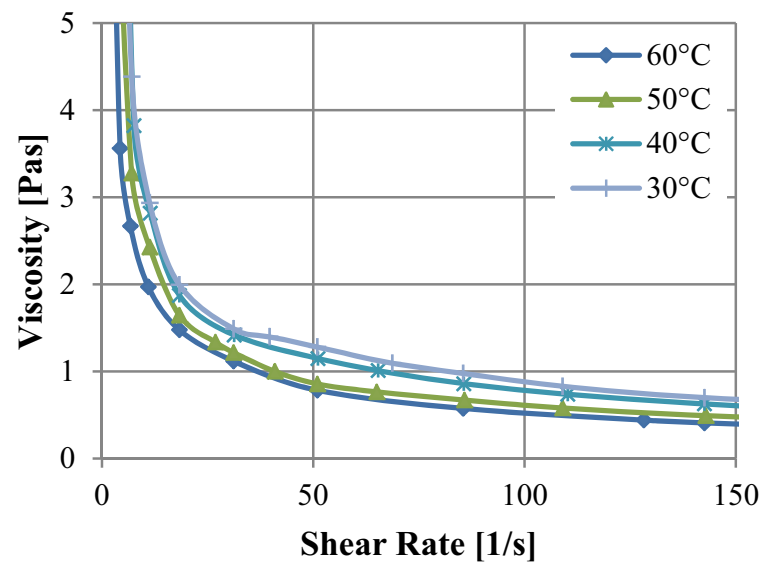

Fig. 3 - Description of the dynamic viscosity in dependent of shear rate from cattle manure with several temperatures (source [4])

In figure 3 it is obvious that cattle manure is like pig manure a shear-thinning substance. The variation of the temperature led to an alteration of the dynamic viscosity. With increasing temperature the dyn. viscosity decreases, see figure 3. The value for the dynamic viscosity is about $3.0 \mathrm{Pas}$ at a shear rate from 1 to $10 \mathrm{l} / \mathrm{s}$. For shear rates above $120 \mathrm{l} / \mathrm{s}$ the viscosity decrease to about $0.5 \mathrm{~Pa}$.

In the following step, cattle and pig manure have to be compared to know how different the viscosity values of these two kinds of slurry are. In order to allow a qualitative comparison of the two manure types, the dry matter content and the temperature of the slurries have to be approximately equal. The dry matter content of the two liquid manures was $11.6 \%-D M$. There was no infor- 
mation about the slurry temperature but both measurements were realized at the same measurement series, so constant boundary conditions are assumed.

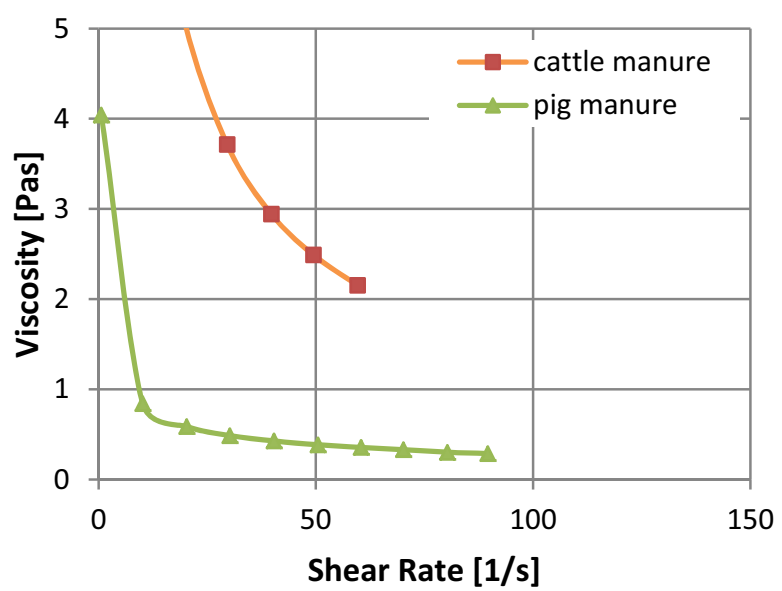

Fig. 4 - Description of the comparison between cattle and pig manure at the same dry matter content (source [3])

It is obvious that the dynamic viscosity of pig manure is lower compared to cattle manure, which is shown in figure 4. Partially the deviation of the viscosity is about $80 \%$.

\section{Conclusion:}

The substitute fluid must be a shear-thinning substance. Furthermore the viscosity values should be about $3.0 \mathrm{Pas}$ at lower shear rates from about 0 to $10 \mathrm{l} / \mathrm{s}$. Higher shear rates (about $2001 / s$ ) require a dynamic viscosity from approximately $0.5 \mathrm{~Pa}$. On the top the fluid must be transparent because a laser Doppler anemometer measurement needs a diaphanous substance.

\section{Examinations of a substitute fluid for cattle and pig manure}

After the mechanical properties are known, the next task is to identify a substitute fluid. The shear-thinning substances which are mentioned in the pre-text can't be used because for a variety of reasons. For example ketchup can't be used because it is not transparent and so it's not possible to use it for a laser Doppler anemometer measurement. Or buttermilk with flakes of butter is a shearthinning medium which has the same mechanical properties (viscosity) [5] like pig or cattle liquid manure. But because of the colour and the perishability buttermilk with flakes of butter can't be used as a substitute medium. In search for another substitute fluid many shearthinning substances were examined. Not only their rheological properties but also some subjective characteristic were important to evaluate the substitute fluids. The subjective criterion includes transparency, the foaming behaviour and the settling of liquids. The following fluid variations were examined for the experiment:

- soap or shampoo

- soap-ethanol-mixture

- water-glue-mixture

- water-soap-mixture
- water-gelatine-mixture

- water-agar-mixture

Through subjective examinations it became apparent that most of the above mentioned fluids are not relevant for the rheological analyses. It was also apparent that the mixture ratios are of great importance. The above mentioned mixture (regardless which mixture ratio the composition has) soap, soap-ethanol and water-soap proved to be unsuitable. The water-gelatine-mixture convinced at the subjective examination. After a long standing time, it led however to a strong odour. That is the reason why it wasn't analysed in rheological studies. The water-gluemixture convinced with very good subjective properties. But after a long period of disuse the fluid hardened and so this mixture is unusable as substitute medium. All subjective evaluation criteria have been complied with a water-agar-mixture. The convinced mixture ratio is $98.8 \%$ water and $1.2 \%$ agar. In the next step this mixture is examined in a rotational viscometer (the results will follow in the article).

\subsection{Substitute fluid - shampoo/soap}

The literature research indicated that shampoo has a similar rheological behaviour such as liquid manure. Therefore a sample of this fluid was examined in a rotational viscometer at the University of Applied Sciences Zwickau. The figure 5 below shows the values of this measurement in comparison to slurry.

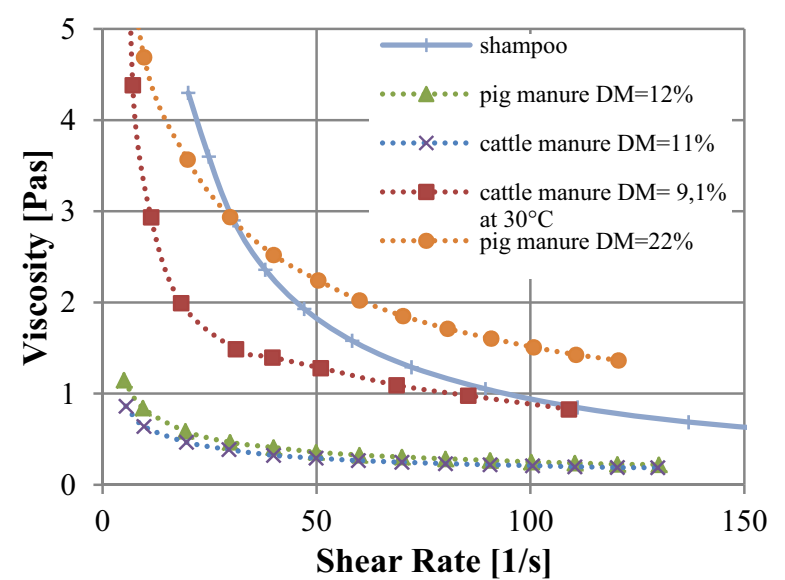

Fig. 5 - Description of the dynamic viscosity form shampoo and some different slurries

As it is apparent in figure 5, the dynamic viscosity values of shampoo is in the range of pig and cattle manure. The examined medium was evaluated subjectively after the rheological analysis. Unfortunately, the subjective investigation led to the conclusion that the pure shampoo or soap can't be used as substitute fluid because it has a bad foaming behaviour. Also a mixture of soap and ethanol can't be used for further investigations because it foams strong too. Furthermore, soap led to strong corrosion on the test rig parts. During a pumping test the soap changed the colour from colourless to yellow resulting from corrosion of the pump. The reason for that is the basic 
character of the soap. The usage of soap as substitute medium is not recommended.

\subsection{Substitute fluid - water-agar-mixture}

The subjective examination of a water-agar-mixture led to good results, so this mixture was analysed with the rotational viscometer. Figure 6 includes the graph of viscosity at different shear rates for the substitute fluid water-agar-mixture.

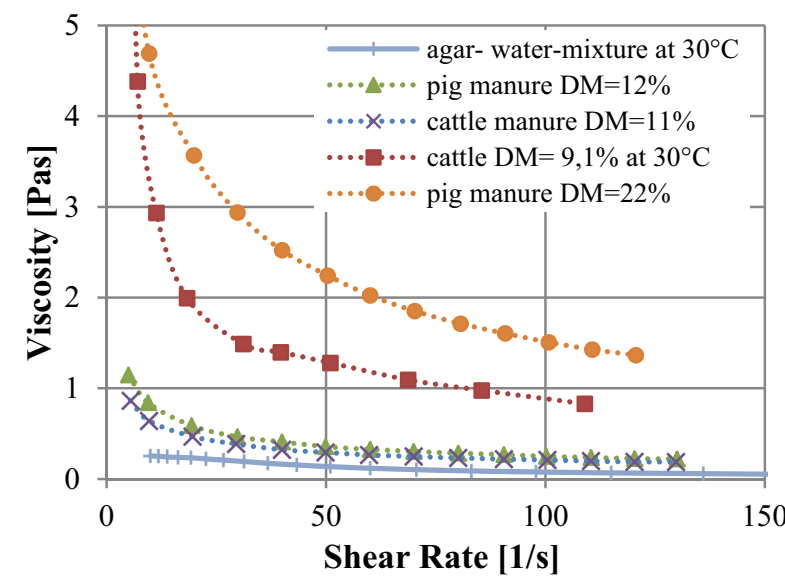

Fig. 6 - Description of the viscosity-course from a water-agarmixture

As it is apparent in figure 6, the water-agar-mixture has a slightly shear-thinning behaviour. At higher shear rates the viscosities of the mixture and pig manure approach each other. To get similarity between the flow of cattle and pig manure with high dry matter content in the distribution, the Reynolds number must be maintained. So the following equation must be complied:

$$
R e_{\text {manure }}=\text { Re }_{\text {substitute fluid }}
$$

A closer look at the Reynolds number equation shows that by changing the velocity and dynamic viscosity at the same liquid manure spreader the dimensionless quantity could be the same.

$$
R e=\frac{v \cdot d \cdot \rho}{\eta}
$$

Through adjusting the velocity at the liquid manure spreader, the mixture of water-agar can represent the characteristics of cattle or pig manure at high shear rates.

\section{Conclusions}

There are some different substitute fluids for pig or cattle manure. The problem decides which substitute fluid is to use. Shampoo and soap has the best mechanical properties compared to slurry. But these two fluids have bad optical properties and they lead to corrosion on metallic components because of their chemical composition. Furthermore shampoo and soap have a bad foaming behaviour. Therefore other substitute fluids were subjective examined. The result is that especially the subjective properties of a water-agar-mixture were advantageous in the case of the problem. Therefore the mixture was analysed in a rotational viscometer. Under the condition of a constant Reynolds number (by changing the flow velocity in the distributor) the properties of the water-agar-mixture and liquid manure is comparable.

\section{Nomenclature}

$\begin{array}{ll}\text { Re } & \text { Reynolds number } \\ \nu & \text { Velocity } \\ d & \text { Characteristic length } \\ \rho & \text { Density } \\ \eta & \text { Dynamic viscosity } \\ \text { dyn. } & \text { Dynamic } \\ \text { DM } & \text { Dry matter } \\ \text { Pas } & \text { Pascal-Seconds } \\ \text { s } & \text { Second's } \\ \text { LDA } & \text { Laser-Doppler-Anemometer }\end{array}$

\section{References}

1. M. Breuer: Laser-Doppler-Anemometrie. Vertiefungspraktikum Strömungsmechanik, 2 (2014)

2. M. Türk.: Das Fließverhalten von trockensubstanzreicher Schweinegülle. agrartechnik, 36, 33 (1986)

3. M. Türk.: Zum Fließverhalten von Güllemischungen. agrartechnik, 37, 64 (1987)

4. H.M. El-Mashad, W.K.P. van Loon, G. Zeeman, G.P.A. Bot: Rheological properties of dairy cattle manure. Bioresource Technology (2004)

5. M. Breitwieser: Fließverhalten von Buttermilch mit Butterflocken. IMETER Methode Nr. 5, (2013) 